\title{
Principal Submatrices of a Full-Rowed Non-Negative Matrix ${ }^{1}$
}

\author{
K. Goldberg
}

(April 2, 1959)

\begin{abstract}
It is shown that one of the principal submatrices of an incidence matrix without zero rows is a permutation matrix. From this an inspection method is described for determining whether a non-negative matrix is nilpotent. Application is made to graph theory.
\end{abstract}

We begin with a necessary flock of definitions. All matrices in this paper are square matrices. The principal submatrices of a matrix are the matrix itself and those submatrices obtained from it by repeatedly striking out a row and the column of the same index. The leading principal submatrices are those obtained by striking out exactly one row and its corresponding column. The principal minors are the determinants of the principal submatrices.

A zero vector is one with every element equal to 0. A full-rowed matrix is one with no zero rows. A nonnegative matrix is one whose elements are all nonnegative real numbers. An incidence matrix is one whose elements are either 0 or 1 . A permutation matrix is an incidence matrix with exactly one 1 in each row and each column. If some power of a matrix vanishes the matrix is called nilpotent. We shall prove:

Theorem. At least one of the principal submatrices of a full-rowed incidence matrix is a permutation matrix.

Corollary 1. If $N$ is a non-negative matrix with a full-rowed principal submatrix of order $m$, then for some positive $r \leq m$ the trace of $N^{r}$ is at least $r \eta^{r}$, where $\eta$ is the least positive element of $N$.

CoRollary 2. Each principal submatrix of a nilpotent non-negative matrix is nilpotent and has both zero rows and zero columns.

Both the theorem and its first corollary hold with "row" replaced by "column". The theorem relates to similar results on systems of distinct representatives. ${ }^{2}$

The main effect of corollary 2 is to provide a simple algorithm for deciding whether or not a non-negative matrix is nilpotent. If it has no zero rows it is not.

\footnotetext{
1 The preparation of this paper was supported (in part) by the Office of Naval

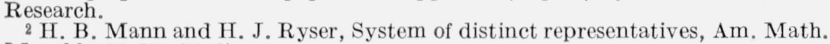
Monthly 60, 397 (1953).
}

Otherwise the zero rows and the columns of the same index are crossed out and the process repeated on the smaller matrix. If none of the matrices examined in this process are full-rowed, then all the principal minors of the matrix are 0 . The characteristic equation is thus of the form $x^{n}=0,{ }^{3}$ and the matrix is nilpotent. R. Marimont ${ }^{4}$ first described this process for incidence matrices.

That the principal submatrices of a nilpotent nonnegative matrix are also nilpotent is known. We have included this statement in corollary 2 because a new proof follows immediately from the remaining result in this corollary.

We prove the theorem by induction. It is certainly true for a matrix of order 1 . Assume that it is true for matrices of order $n-1$.

Let $A=\left(a_{i j}\right)$ be a full-rowed incidence matrix of order $n$. If any of its leading principal submatrices is full-rowed then we are finished because "principal submatrix" is a hereditary property. Therefore, we assume that each of the leading principal submatrices of $A$ has at least one zero row. We shall prove that then $A$ is a permutation matrix.

Let $A_{s}$ denote the leading principal submatrix of $A$ obtained by striking out the row and column of index $s$. We may assume that there is an index $i_{s}$ such that

$$
a_{i_{s} j}=0 \text { for } j=1,2, \ldots, s-1, s+1, \ldots, n .
$$

Since no row of $A$ is a zero row this implies that

$$
a_{i_{s} s}=1 \text {. }
$$

If another leading principal submatrix $A_{t}$ also had as one of its zero rows a row corresponding to the index $i_{s}$ we would have $a_{i_{s} t}=1$ with $t \neq s$. This

3 C. C. MacDuffee, The theory of matrices, p. 19 (Chelsea Pub. Co., New York, N.Y., 1946).

$4 \mathrm{R}$. Marimont, A new method of checking the consistency of precedence matrices, J. Assoc. Computing Machinery 6, 164 (1959). 
would contradict (2.1). Therefore, to each $s$ there corresponds a unique $i_{s}$ which satisfies (2.1) and (2.2). It follows that $A$ is a permutation matrix, and the theorem is proved.

For any non-negative matrix $N$ let $\bar{N}$ denote the incidence matrix of the same order as $N$, with 1 in every position that $N$ has a positive element and 0 in every position that $N$ has 0 . If $\eta$ is the least positive element in $N$ then $N-\eta \bar{N}$ is a non-negative matrix. Thus, if $\operatorname{tr} X$ denotes the trace of $X$,

$$
\operatorname{tr} N^{r} \geq \eta^{r} \operatorname{tr} \bar{N}^{r}
$$

for every non-negative integer $r$.

Furthermore, let $\mathrm{N}=\left(n_{i j}\right)$ be of order $n$, let $S=\{1,2, \ldots, n\}$, and let $S_{0}$ be any subset of $S$. Let $M$ be the principal submatrix of $N$ with row and column indices in $S_{0}$. Then for any positive integer $r$ we have

$$
\begin{aligned}
\operatorname{tr} N^{r}=\sum_{i_{i} \in S} n_{i_{1} i_{2}} n_{i_{2} i_{3}} \ldots & n_{i_{r} i_{1}} \\
& \geq \sum_{i_{j} \in S_{0}} n_{i_{1} i_{2}} n_{i_{2} i_{3}} \ldots n_{i_{r} i_{1}}=\operatorname{tr} M^{r} .
\end{aligned}
$$

Now suppose $M$ is a full-rowed principal submatrix of $N$ of order $m$. Then $\bar{M}$ is a full-rowed incidence matrix, and so has a principal submatrix $P$ of order $s \leq \mathrm{m}$ which is a permutation matrix. Let $r \leq S$ be any of the cycle lengths of the permutation corresponding to $\dot{P}$. Then

$$
\operatorname{tr} P^{r} \geq r
$$

Since $P$ is a principal submatrix of $\bar{M}$, the incidence matrix corresponding to $M$, and $M$ is a principal submatrix of $N$, we have

$$
\operatorname{tr} N^{r} \geq \operatorname{tr} M^{r} \geq \eta^{r} \operatorname{tr} \overline{M^{r}} \geq \eta^{r} \operatorname{tr} P^{r} \geq r \eta^{r}
$$

with $r \leq s \leq m$. This proves corollary 1 .

Corollary $\overline{2}$ follows directly from corollary 1 and the fact that a matrix is nilpotent (if and) only if the traces of its positive powers are all 0 .

Our theorem can also be stated as a result on directed graphs. A directed graph G is a set of points $P=\{p, q, \ldots\}$ and a subset $P^{*}=\{(p, q), \ldots\}$ of the Cartesian product $P \times P$. The ordered pair $(p, q)$ may be thought of as a directed line from $p$, called the starting point, to $q$.

We say that $G$ contains a simple loop if there is a subset $P^{\prime}=\left\{p_{1}^{\prime}, p_{2}^{\prime}, \ldots, p_{n}^{\prime}\right\}$ of $P$ such that

$P^{*} \cap\left(P^{\prime} \times P^{\prime}\right)=\left\{\left(p_{1}^{\prime}, p_{2}^{\prime}\right),\left(p_{2}^{\prime}, p_{3}^{\prime}\right), \ldots\right.$,

$$
\left(p_{n-1}^{\prime}, p_{n}^{\prime}\right),\left(p_{n}^{\prime}, p_{1}^{\prime}\right\} \text {. }
$$

That is to say, the points of $P^{\prime}$ have these lines between them, and no others.

Our theorem is equivalent to the following statement: If every point of a directed graph is a starting point then the graph contains a simple loop.

As so stated, this theorem is essentially one proved by David Rosenblatt. ${ }^{5}$

${ }^{5} \mathrm{D}$. Rosenblatt, On the graphs and asymptotic forms of finite Boolean relation matrices and stochastic matrices, Naval Res. Log. Quart. 4, 151 (1957).

Washington, D.C.

(Paper 63B1-3) 